Originalien

Unfallchirurg $2020 \cdot 123: 792-796$

https://doi.org/10.1007/s00113-020-00788-5

Online publiziert: 25 . Februar 2020

(c) Der/die Autor(en) 2020

\section{Redaktion}

W. Mutschler, München

H. Polzer, München

B. Ockert, München

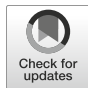

H. Erne' $\cdot$ P. Moog $\cdot$ M. Cerny' $\cdot$ S. Loew ${ }^{2} \cdot$ J. Betzl' $\cdot$ H.-G. Machens ${ }^{1} \cdot$ D. Schmauss ${ }^{3}$

'Abteilung für Plastische Chirurgie und Handchirurgie, Klinikum rechts der Isar, Technische Universität München, München, Deutschland

${ }^{2}$ Praxis für Handchirurgie und Traumatologie, Bad Mergentheim, Deutschland

${ }^{3}$ Abteilung für Plastische, Rekonstruktive und Ästhetische Chirurgie, Ospedale Regionale di Lugano, Lugano, Schweiz

\title{
Genauigkeit der konventionellen Röntgendiagnostik bei der Beurteilung der Daumensattelgelenkarthrose
}

\section{Einleitung}

Die Arthrose des Daumensattelgelenks (CMC I) ist eine häufige Pathologie der Hand, die Schmerzen verursacht und in späten Stadien zu einer deutlich reduzierten Beweglichkeit im Daumensattelgelenk führen kann [1]. Das diagnostische Vorgehen besteht meist aus Anamnese, klinischer Untersuchung sowie konventioneller Radiographie des Daumensattelgelenks in 2 Ebenen. Die Ausdehnung der Arthrose im radiologischen Befund wird dabei in Stadien nach Eaton und Littler klassifiziert [2].

In den letzten Jahren spielt die Arthroskopie des Daumensattelgelenks eine zunehmend größere Rolle sowohl bei der Diagnose als auch bei therapeutischen Interventionen im Rahmen der symptomatischen Arthrose [3, 4]. Wie auch bei anderen Arthroskopien kann der Knorpel so direkt dargestellt, mittels Sondierung untersucht und therapeutische Gelenklavagen, Synovialektomie und andere Interventionen können durchgeführt werden. Die Schwere der Arthrose in einer Arthroskopie wird dabei unabhängig vom Gelenktyp nach der OuterbridgeKlassifikation angegeben (• Tab. 1).

Nachdem in den letzten Jahren zahlreiche Patienten mittels Daumensattelgelenkarthroskopie behandelt wur-

H. Erne und P. Moog haben in gleichem Maße zur Entstehung dieser Arbeit beigetragen. den, musste festgestellt werden, dass die Ausprägung der Knorpeldefekte in der Arthroskopie oft vom präoperativen röntgenologischen Schweregrad abweicht. Da es jedoch bisher in der Literatur keine konkreten Daten dazu gibt, zielt diese Studie darauf ab, die diagnostische Exaktheit von konventionellen Röntgenuntersuchungen im Vergleich zur Arthroskopie bei Daumensattelgelenkarthrose $\mathrm{zu}$ evaluieren.

Zudem wird postuliert, dass die $\mathrm{Ou}$ terbridge-Klassifikation, die üblicherweise zur Klassifikation der Knorpelbeschaffenheit am Kniegelenk verwendet wird, nicht zur Anwendung im Bereich des Daumensattelgelenks geeignet ist, da sie auf Parametern wie der Knorpeldicke basiert, die bei einem solch kleinen Gelenk nur schwer beurteilbar sind. Nichtsdestotrotz wird die EatonLittler-Röntgenklassifikation oftmals in die Outerbridge-Knorpelklassifikation übertragen. Daraus werden Rückschlüsse für die Therapie gezogen, ohne Kenntnis über die Knorpelbeschaffenheit zu besitzen. Aufgrund dieser inkorrekten Übertragung haben wir eine spezifische Klassifikation für den Schweregrad der Daumensattelgelenkarthrose entwickelt, sowie das bereits für andere Gelenke, die ebenso fälschlicherweise die kniespezifische Outerbridge-Klassifikation genutzt haben, umgesetzt wurde. Wir vermuten, dass die diagnostische Genauigkeit des Knorpelschadens im Daumensattelge- lenk im konventionellen Röntgen sehr gering ist und daher ein Bedarf für eine spezifische arthroskopische Klassifikation des Daumensattelgelenks besteht.

\section{Material und Methoden}

Zwischen 2015 und 2017 wurden 23 Arthroskopien des Daumensattelgelenks bei 23 Patienten mit symptomatischer Daumensattelgelenkarthrose der Stadien 1 bis 4 nach Eaton und Littler durchgeführt. Alle Arthroskopien wurden von demselben Facharzt für Handchirurgie vorgenommen, wobei die Schweregrade in den jeweiligen Befunden nach Outerbridge klassifiziert wurden. Die präoperativen konventionellen Röntgenbilder der 23 Patienten - allesamt von guter und repräsentativer Qualität - wurden anschließend retrospektiv gesammelt und von $10 \mathrm{er}$ fahrenen Fachärzten für Handchirurgie, die nicht in die Therapie involviert waren, beurteilt. Den Untersuchern, welche gegenüber den Arthroskopiebefunden verblindet waren, wurde bei Beurteilung der konventionellen Röntgenbilder folgende Frage gestellt: „Welches Arthrosestadium nach Outerbridge würden Sie in der Arthroskopie erwarten?" Die Antworten wurden anschließend mit den in der Arthroskopie festgestellten Outerbridge-Stadien verglichen, welche aus den Patientenakten erhoben wurden. 


\begin{tabular}{|c|c|}
\hline Stadium & Arthroskopie Befunde \\
\hline 0 & Normaler Knorpelstatus \\
\hline I & Knorpelerweichung und Schwellung bei erhaltener Knorpeloberfläche \\
\hline II & $\begin{array}{l}\text { Aufgefaserte Knorpeloberfläche mit Fissuren, die } 50 \% \text { der Knorpeloberfläche nicht } \\
\text { überschreiten }\end{array}$ \\
\hline III & $\begin{array}{l}\text { Aufgefaserte Knorpeloberfläche über } 50 \% \text {, subchondraler Knochen noch nicht } \\
\text { erreicht }\end{array}$ \\
\hline IV & Vollschichtiger Knorpelverlust \\
\hline
\end{tabular}

Tab. 2 Beurteilung der Daumensattelgelenkarthrose in der Arthroskopie (nach Outerbridge) unserer 23 Patienten im Vergleich zur konventionellen Röntgendiagnostik hinsichtlich der Genauigkeit in der Stadieneinteilung bei isolierter Betrachtung der konventionellen Röntgenbefunde

\begin{tabular}{|l|l|l|}
\hline $\begin{array}{l}\text { Outerbridge- } \\
\text { Klassifikation }\end{array}$ & $\begin{array}{l}\text { Anzahl der Patienten, die } \\
\text { mittels Arthroskopie bewer- } \\
\text { tet wurden }\end{array}$ & $\begin{array}{l}\text { Prozentsatz der korrekten Stadieneintei- } \\
\text { lung unter isolierter Betrachtung der konv. } \\
\text { Röntgenaufnahmen }\end{array}$ \\
\hline I & 0 & - \\
\hline II & 4 & $13 \%(95 \%-K I ~ 3-23 \%)$ \\
\hline III & 8 & $38 \%(95 \%-K I ~ 27-49 \%)$ \\
IV & 11 & $73 \%(95 \%-K I ~ 65-81 \%)$ \\
\hline 95\%-KI 95\% Konfidenzintervall &
\end{tabular}

\section{Tab. 3 Arthroskopieklassifikation der Daumensattelgelenkarthrose}

\begin{tabular}{ll}
$\begin{array}{l}\text { Stadien- } \\
\text { einteilung }\end{array}$ & Arthroskopie Befunde \\
\hline Stadium I & Synovitis ohne Knorpelschaden \\
\hline $\begin{array}{l}\text { Stadium II } \\
\text { Stadium III }\end{array}$ & $\begin{array}{l}\text { Deutliche fokale Knorpelläsionen, die nicht größer als die Sondenspitze sind } \\
\text { unvollständig) }\end{array}$ \\
\hline Stadium IV & \begin{tabular}{l} 
Kein Knorpel mehr im belastenden Bereich, nur noch kleine Knorpelrandstreifen \\
\hline
\end{tabular}
\end{tabular}

\section{Operationstechnik}

Die Arthroskopie des Daumensattelgelenks wird über 2 Standardzugänge, das 1R- und das 1U-Portal, durchgeführt [3, 4]. Das 1R-Portal befindet sich radial der Sehne des M. abductor pollicis longus (APL), das 1U-Portal ulnar der Sehne des M. extensor pollicis brevis (EPB) [1].

Ein weiteres radiales Portal kann in der Verlängerung der Sehne des M. flexor carpi radialis (FCR) geschaffen werden, direkt oberhalb des Daumensattelgelenks $[5,6]$. Dieses sog. Thenar-Portal steht über dem Thenar im rechten Winkel $\left(90^{\circ}\right)$ zum 1U-Portal [7]. Es wird vom 1U-Portal aus mittels Diaphanoskopie erreicht, was sich aufgrund des sehr engen Raums allerdings schwierig gestalten kann. Dieser Zugang kann durch eine fluoroskopiekontrollierte Kanülierung erleichtert werden. Nach intraartikulärer Platzierung der Kanüle kann grenzenden Bänder sowie der ulnaren Anteile des palmaren Gelenkkomplexes. Um die Beurteilung der Gelenksstrukturen zu verbessen, werden die Instrumente mindestens einmal zwischen $1 \mathrm{U}$ und $1 \mathrm{R}$ ausgetauscht. Falls ein Shaving durchgeführt wurde, wird die Arthroskopie mit der Injektion eines Gemisches aus Steroiden und Lokalanästhetika beendet. Die Portale bleiben offen und werden nur mit sterilen Pflasterstreifen versorgt. Es wird ein steriler Wundverband angelegt und eine palmare Daumenschiene angepasst. Wundkontrollen und Verbandwechsel finden am ersten postoperativen Tag statt.

\section{Ergebnisse}

Die Stadien der Arthrose, die in den Arthroskopien der 23 Patienten gefunden wurden, sowie den Anteil der korrekt beurteilten dazugehörigen konventionellen Röntgenbilder zeigt - Tab. 2.

Von den 23 Daumensattelgelenken hatten 11 Knorpeldefekte 4 . Grades nach Outerbridge, 8 Läsionen 3. Grades, 4 Läsionen 2. Grades und keiner Läsionen 1. Grades.

Insgesamt wurden $43 \% \quad$ (95\%-KI $37-49 \%)$ der Knorpeldefekte unter ausschließlicher Verwendung von röntgenologischen Kriterien richtig diagnostiziert. Bei den Grad-4-Läsionen stellten 73\% (95\%-KI 65-81\%) der Untersucher die richtige Diagnose, bei denen 3. Grades waren es $38 \%$ (95\%-KI 27-49\%) und bei denen 2. Grades $13 \%$ (95\%-KI 3-23\%). Bei jeder Fehleinschätzung wurde der Schweregrad der Arthrose unterschätzt, nie jedoch überschätzt.

\section{Diskussion}

die Gelenkkapsel mit der stumpfen Schere perforiert, der Trokar in den Gelenkspalt platziert und das 1,9-mm-Arthroskop eingeführt. Im Fall einer ausgeprägten Synovitis kann ein Débridement mittels „shaver“ notwendig werden, um die intraartikulären Strukturen besser darstellen zu können.

Die Artikulation wird mit einer Sonde navigiert, welche über das 1U-Portal eingeführt wird. Das 1R-Portal erlaubt die Untersuchung der dorsalen und ulnaren Anteile der Gelenkfläche, der an-
In der klinischen Routine wird das Daumensattelgelenk meist mittels konventioneller Röntgenaufnahme evaluiert, wobei der Schweregrad nach Eaton und Littler klassifiziert wird [2]. In Spezialfällen wird zudem ggf. eine zusätzliche Magnetresonanztomographie (MRT) zu Diagnosesicherung und Feststellung der Knorpelbeschaffenheit und der Kapsel-/ Bandläsionen durchgeführt [1]. Als Grundlage jeder Therapieentscheidung steht jedoch die klinische Symptomatik 
Unfallchirurg 2020 · 123:792-796 https://doi.org/10.1007/s00113-020-00788-5

(c) Der/die Autor(en) 2020

\section{H. Erne $\cdot$ P. Moog $\cdot$ M. Cerny $\cdot$ S. Loew $\cdot$ J. Betzl $\cdot$ H.-G. Machens $\cdot$ D. Schmauss \\ Genauigkeit der konventionellen Röntgendiagnostik bei der Beurteilung der Daumensattelgelenkarthrose}

\section{Zusammenfassung}

Hintergrund. Die Daumensattelgelenkarthrose ist ein häufiges und schmerzhaftes Krankheitsbild, das mittels konventioneller Röntgenbildgebung und Arthroskopie diagnostiziert und ggf. therapiert werden kann. Dabei stellt die Röntgenklassifikation oft den arthroskopisch dargestellten Knorpelschaden nicht angemessen dar.

Ziel der Arbeit (Fragestellung). Das Ziel dieser Arbeit war, die Genauigkeit von konventioneller Röntgendiagnostik im Vergleich zu Arthroskopie bei Daumensattelgelenkarthrose zu beurteilen.

Material und Methoden. Die präoperativen konventionellen Röntgenbilder von 23 Patienten wurden 10 erfahrenen Handchirurgen vorgelegt, die gegenüber den dazugehörigen
Arthroskopiebefunden verblindet waren. Ihre Beurteilungen wurden anschließend mit den Befunden der Arthroskopien verglichen. Ergebnisse. Elf Patienten wiesen Läsionen 4. Grades nach der Outerbridge-Klassifikation auf, 8 Läsionen 3. Grades, 4 Läsionen 2. Grades und keiner Läsionen 1. Grades. Insgesamt wurden $43 \%$ (95\%-KI[Konfidenzintervall] 37-49\%) der Knorpelläsionen mittels konventionellen Röntgens korrekt diagnostiziert. Für die Läsionen 4. Grades stellten 73 \% (95\%-KI 65-81\%) der Untersucher korrekte Diagnosen, für die 3. Grades 38\% (95\%-KI 27-49\%) und für die 2. Grades $13 \%$ (95\%-KI 3-23\%). Schlussfolgerung. Diese Studie präsentiert ein arthroskopisches Klassifikationssystem, das spezifisch für die Daumensattelgelenkarthrose ist und eine weitere Beurteilungsmöglichkeit darstellen kann, falls die Bilder für die röntgenologischen Klassifikation nach Eaton und Littler zu unspezifisch sind. Hier kann die Arthroskopie eine genauere Einteilung in therapierelevante Stadien ermöglichen und umfasst zudem auch therapeutische Optionen.

\section{Schlüsselwörter}

Daumensattelgelenkarthrose - Handgelenkarthroskopie · Outerbridge-Klassifikation · Eatonund-Littler-Klassifikation · Konventionelle Röntgenbildgebung

\section{Diagnostic accuracy of conventional X-ray imaging for assessment of trapeziometacarpal joint osteoarthritis}

\section{Abstract}

Background. Osteoarthritis of the trapeziometacarpal joint is a common and painful affliction that can be diagnosed using conventional X-ray imaging as well as arthroscopy and if necessary treated; however, the X-ray classification often does not sufficiently demonstrate the cartilage damage found in arthroscopy.

Objective. The aim of the study was to evaluate the diagnostic accuracy of conventional X-ray imaging compared to arthroscopy for osteoarthritis of the trapeziometacarpal joint. Material and methods. The preoperative conventional X-ray images of 23 patients were presented to 10 experienced hand surgeons who were blinded to the arthroscopy results. Their ratings were compared to the results found with arthroscopy.

Results. Of the patients 11 were found to have grade 4 cartilage lesions according to the Outerbridge classification, 8 grade 3 lesions, 4 grade 2 lesions and no grade 1 lesions. Overall, 43\% (95\% confidence interval, Cl 37-49\%) of the cartilage lesions were diagnosed correctly using conventional $\mathrm{X}$-rays. For grade 4 lesions $73 \%(95 \% \mathrm{Cl} 65-81 \%)$ of the surgeons made the correct diagnosis, in the case of grade 3 lesions $38 \%(95 \% \mathrm{Cl}$ $27-49 \%)$ and in grade 2 lesions $13 \%(95 \% \mathrm{Cl}$ 3-23\%).
Conclusion. This study presents an arthroscopy classification system that is specific for osteoarthritis of the trapeziometacarpal joint and provides an additional method of assessment if the $\mathrm{X}$-ray grading of osteoarthritis according to Eaton and Littler is too unspecific. Arthroscopy can be used to safely differentiate the treatment-relevant stage and also includes treatment options.

\section{Keywords}

Trapeziometacarpal joint osteoarthritis - Wrist arthroscopy - Outerbridge classification - Eaton and Littler · X-ray des Patienten. Goldstandard in der operativen Therapie der fortgeschrittenen symptomatischen Daumensattelgelenkarthrose (Stadien III und IV) ist die Resektionsarthroplastik. Jedoch ist dieser Ansatz mit mittel- bis langfristigen Komplikationen wie der Proximalisation des Daumens und einer langen Rekonvaleszenzdauer verbunden [8-14].

Daher spielt die Arthroskopie des Daumensattelgelenks eine zunehmend wichtige Rolle bei Patienten mit Leidensdruck, die röntgenologisch dem Stadium
I, II oder III nach Eaton und Littler zugeordnet werden können. Hier erlaubt die Arthroskopie eine eingehendere Diagnostik und die direkte therapeutische Intervention [3, 4], wie z.B. „lipofilling“, Denervation, Entfernung eines freien Gelenkkörpers oder Gelenklavage, die einfach in eine diagnostische Arthroskopie eingebettet werden können.

Unsere Studie zeigt, dass die Auswertung von konventionellen Röntgenbildern während des diagnostischen Prozesses bei Daumensattelgelenkarthrose zu einer Fehleinschätzung des Schweregrads in $57 \%$ der Fälle führen kann, wenn man sie mit den arthroskopischen Befunden vergleicht. Dies war von der Ausprägung der Arthrose abhängig, sodass schwerwiegendere Befunde genauer diagnostiziert wurden als weniger schwere.

Insgesamt scheint die röntgenologische Befundung jedoch den Schweregrad zu unterschätzen. Daher sollte die Schwelle zu invasiver Diagnostik und Therapie bei symptomatischen Patienten niedrig sein. Es darf nämlich auch 


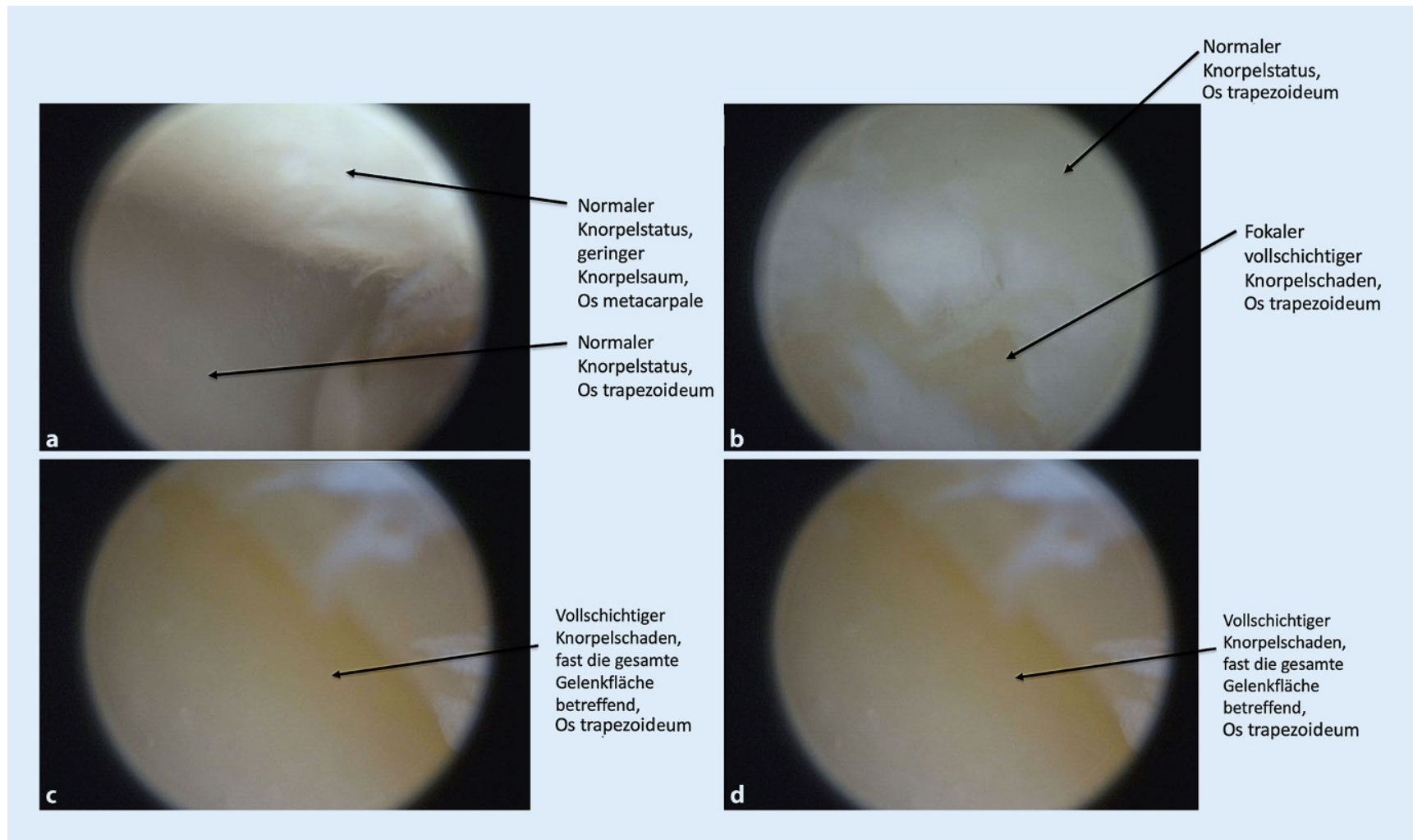

Abb. 1 ॥ Neue Arthroskopieklassifikation der Daumensattelgelenkarthrose anhand von Arthroskopie-Bildern. a Stadium I, b Stadium II, c Stadium III, d Stadium IV

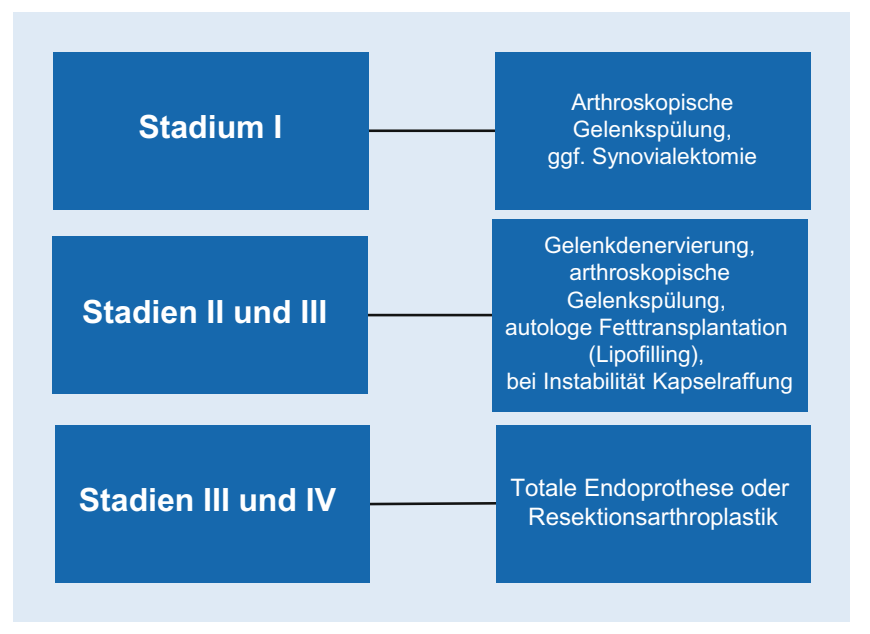

Abb. $2<$ Algorithmus für die Therapie der Daumensattelgelenkarthrose, Stadieneinteilung nach der neuen Arthroskopieklassifikation (•Tab. 3; - Abb. 1) nicht vergessen werden, dass diese diagnostische Fehlinterpretation durchaus Einfluss auf die Therapieentscheidung haben kann.

Zudem postulieren wir, dass die $\mathrm{Ou}$ terbridge-Klassifikation zur Beurteilung von Athroskopiebefunden des Daumensattelgelenks nicht ausreichend ist, da diese zur Beurteilung kleiner Gelenke ungeeignet ist. Stadien II und III werden anhand der Knorpeldicke eingeteilt, mehr bzw. weniger als $50 \%$ der Ausgangsdicke. Unserer Ansicht nach ist es erschwert, dies am Daumensattelgelenk sicher zu beurteilen, da hier die Knorpelschicht generell sehr dünn ist. Außerdem können die Defekte sich auch in frühen Stadien schon auf die gesamte Knorpeldicke ausdehnen, wenn nur kleine Bereiche betroffen sind, wobei deren Größe das ausschlaggebende Kriterium zu sein scheint. Die arthroskopische Klassifikati- on der Daumensattelgelenkarthrose nach Badia dürfte ebenfalls zu grob sein, da sie nur 3 Stadien unterscheidet [15]. In unserer Patientenklientel bestanden große Unterschiede bezüglich Läsionen des Stadiums II nach Badia, welcher „fokale Knorpeldefekte" beschreibt.

Aufgrund unserer Beobachtungen und der Limitationen der bestehenden arthroskopischen Klassifikationssysteme schlagen wir deshalb ein neues Klassifikationssystem vor (• Tab. 3; • Abb. 1).

Prinzipiell sollten alle symptomatischen Patienten mit Daumensattelgelenkarthrose, egal welchen Eaton-und-Littler-Stadiums, mittels konventionellem Röntgen untersucht werden. Allerdings gewinnt man durch die röntgenologische Untersuchung hauptsächlich Informationen anhand des Subluxationsgrades zu der Gelenksstabilität, während die Arthroskopie den Knorpelschaden akkurater darstellen kann. Die Kombination dieser diagnostischen Herangehensweisen kann neue Erkenntnisse ermöglichen. Daher kann zusammenfassend festgestellt werden, dass beide diagnostischen Mittel sinnvoll sind: die Arthroskopie 
für die Beurteilung des Arthrosegrads anhand des Knorpelzustands und das konventionelle Röntgen für die Gelenkstabilität. Ergänzt wird dies durch die klinische Untersuchung, die Auskunft über das Ausmaß der Gelenkinstabilität und der Alltagseinschränkung gibt.

Auch im Fall, dass Unsicherheiten oder Differenzen zwischen klinischem Bild und Röntgenklassifikation bestehen, sollte eine zusätzliche Arthroskopie durchgeführt werden. Insbesondere bei grenzwertigen Fällen zwischen Stadien II und III nach Eaton und Littler sollten alle verfügbaren diagnostischen Ressourcen ausgeschöpft werden, bevor die Indikation zur Resektionsarthroplastik gestellt wird. Unser Vorschlag bezüglich eines Therapiealgorithmus im Rahmen unseres spezifischen Klassifikationssystems wird daher in • Abb. 2 gezeigt.

\section{Limitationen}

Eine Schwäche unserer Studie besteht darin, dass keiner der Patienten eine arthroskopisch gesicherte Daumensattelgelenkarthrose 1. Grades nach Eaton und Littler hat. Jedoch sind diese Patienten meist klinisch asymptomatisch und werden daher nicht arthroskopiert.

\section{Fazit für die Praxis}

Die Beurteilung der Knorpelläsionen am Daumensattelgelenk mittels konventioneller Röntgenaufnahme ist nicht ausreichend. Die Arthroskopie kann zur Abschätzung von Knorpeldefekten und zur Prognosevorhersage eine sinnvolle Ergänzung darstellen und sollte daher zusätzlich in der Routinediagnostik verwendet werden, besonders bei Unsicherheiten oder Diskrepanzen zwischen klinischem Bild und radiologischem Befund.

Unser arthroskopisches Klassifikationssystem und unser Behandlungsalgorithmus sollten die Diagnose und Therapie der Daumensattelgelenkarthrose verbessern. Wir empfehlen, diese Herangehensweise in zukünftigen Studien weitergehend zu evaluieren.

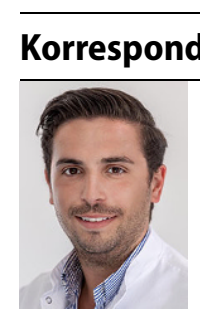

\section{Dr. med. P. Moog}

Abteilung für Plastische

Chirurgie und Handchirurgie, Klinikum rechts der Isar,

Technische Universität

München

Ismaninger Str. 22,

81675 München, Deutschland philippmoog@web.de

Funding. Open Access funding provided by Projekt DEAL.

\section{Einhaltung ethischer Richtlinien}

Interessenkonflikt. H. Erne, P. Moog, M. Cerny, S. Loew, J. Betzl, H.-G. Machens und D. Schmauss geben an, dass kein Interessenkonflikt besteht. Diese Studie hat keinerlei finanzielle Unterstützung von öffentlichen, kommerziellen oder Non-Profit-Organisationen erhalten.

Für diesen Beitrag wurden von den Autoren keine Studien an Menschen oder Tieren durchgeführt. Für die aufgeführten Studien gelten die jeweils dort angegebenen ethischen Richtlinien.

Open Access Dieser Artikel wird unter der Creative Commons Namensnennung 4.0 International Lizenz veröffentlicht, welche die Nutzung, Vervielfältigung, Bearbeitung, Verbreitung und Wiedergabe in jeglichem Medium und Format erlaubt, sofern Sie den/die ursprünglichen Autor(en) und die Quelle ordnungsgemäß nennen, einen Link zur Creative Commons Lizenz beifügen und angeben, ob Änderungen vorgenommen wurden.

Die in diesem Artikel enthaltenen Bilder und sonstiges Drittmaterial unterliegen ebenfalls der genannten Creative Commons Lizenz, sofern sich aus der Abbildungslegende nichts anderes ergibt. Sofern das betreffende Material nicht unter der genannten Creative Commons Lizenz steht und die betreffende Handlung nicht nach gesetzlichen Vorschriften erlaubt ist, ist für die oben aufgeführten Weiterverwendungen des Materials die Einwilligung des jeweiligen Rechteinhabers einzuholen.

Weitere Details zur Lizenz entnehmen Sie bitte der Lizenzinformation auf http://creativecommons.org/ licenses/by/4.0/deed.de.

\section{Literatur}

1. Kalb K (2014) Die Arthroskopie des Daumensattelgelenks. Oper Orthop Traumatol 26:556-563

2. Eaton RG, Lane LB, Littler JW, Keyser JJ (1984) Ligament reconstruction for the painful thumb carpometacarpal joint: a long-term assessment. JHand Surg Am 9:692-699

3. Berger RA (1997) A technique for arthroscopic evaluation of the first carpometacarpal joint. JHand Surg Am 22:1077-1080

4. Menon J (1996) Arthroscopic management of trapeziometacarpal joint arthritis of the thumb. Arthroscopy 12:581-587
5. Carro LP, Golano P, Vega J et al (2006) Innovations in arthroscopic management of basal joint arthritis of the thumb. Arthroscopy 22:1361-1364

6. Orellana MA, Chow JC (2003) Arthroscopic visualization of the thumb carpometacarpal joint: introduction and evaluation of a new radial portal. Arthroscopy 19:583-591

7. Walsh EF, Akelman E, Fleming BC, da Silva MF (2005) Thumb carpometacarpal arthroscopy: a topographic, anatomic study of the thenar portal. JHand Surg Am 30:373-379

8. Ehrl D, Erne HC, Broer PN, Metz C, Falter E (2016) Painful thumb carpometacarpal joint osteoarthritis: results of a novel treatment approach. J Plast Reconstr Aesthet Surg 69:972-976

9. Ehrl D, Erne HC, Broer PN, Metz C, Falter E (2016) Outcomes of denervation, joint lavage and capsular imbrication for painful thumb carpometacarpal joint osteoarthritis. J Hand Surg EurVol 41:904-909

10. Sigfusson R, Lundborg G (1991) Abductor pollicis longus tendon arthroplasty for treatment of arthrosis in the first carpometacarpal joint. Scand J Plast Reconstr Surg Hand Surg 25:73-77

11. Wulle C (1993) Die Abductor pollicis longus-Plastik zur Behandlung der Daumensattelgelenkarthrose. Handchir Mikrochir Plast Chir 25:250-255

12. Eaton RG, Lane LB, Littler JW, Keyser JJ (1984) Ligament reconstruction for the painful thumb carpometacarpal joint: a long-term assessment. JHand Surg Am 9:692-699

13. Ulrich-Vinther M, Puggaard H, Lange B (2008) Prospective 1-year follow up study comparing joint prosthesis with tendon interposition arthroplasty in treatment of trapeziometacarpal osteoarthritis. JHand Surg Am 33:1369-1377

14. Erne H, Cerny MK, Ehrl D, Bauer AT, Schmauss V, Moog P, Broer PN, Loew S, Schmauss D (2018) Autologous fat injection vs. Lundborg's resection arthroplasty for the treatment of trapeziometacarpal joint osteoarthritis. Plast Reconstr Surg 141(1):119-124

15. Badia A (2006) Trapeziometacarpal arthroscopy: a classification and treatment algorithm. Hand Clin 22:153-163 